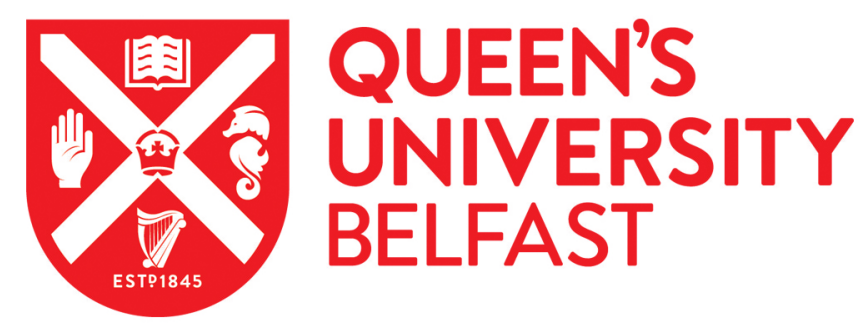

\title{
Translating Mechanism-Based Strategies to Break the Obesity- Cancer Link: A Narrative Review
}

\author{
Smith, L. A., O'Flanagan, C. H., Bowers, L. W., Allott, E. H., \& Hursting, S. D. (2018). Translating Mechanism- \\ Based Strategies to Break the Obesity-Cancer Link: A Narrative Review. Journal of the Academy of Nutrition \\ and Dietetics, 118(4), 652-667. https://doi.org/10.1016/j.jand.2017.08.112
}

Published in:

Journal of the Academy of Nutrition and Dietetics

Document Version:

Peer reviewed version

Queen's University Belfast - Research Portal:

Link to publication record in Queen's University Belfast Research Portal

Publisher rights

(c) 2018 The Academy of Nutrition and Dietetics.

This manuscript version is made available under the CC-BY-NC-ND 4.0 license http://creativecommons.org/licenses/by-nc-nd/4.0/,which permits distribution and reproduction for noncommercial purposes, provided the author and source are cited

\section{General rights}

Copyright for the publications made accessible via the Queen's University Belfast Research Portal is retained by the author(s) and / or other copyright owners and it is a condition of accessing these publications that users recognise and abide by the legal requirements associated with these rights.

Take down policy

The Research Portal is Queen's institutional repository that provides access to Queen's research output. Every effort has been made to ensure that content in the Research Portal does not infringe any person's rights, or applicable UK laws. If you discover content in the Research Portal that you believe breaches copyright or violates any law, please contact openaccess@qub.ac.uk. 
1 Hungering for New Mechanism-Based Strategies to Break the Obesity-Cancer Link:

2 A Narrative Review

\section{$4 \quad$ Research Snapshot}

5 Research Question: What are the mechanisms through which obesity increases cancer

6 risk and progression? Does implementation of dietary or lifestyle interventions attenuate

7 obesity-associated cancer risk factors?

9 Key Findings: A traditional literature review revealed that obesity-associated metabolic

10 perturbations are emerging as major drivers of obesity-related cancer including

11 alterations in growth factor signaling, inflammation and angiogenesis. Preclinical

12 evidence suggests that dietary interventions such as calorie restriction, intermittent

13 fasting, ketogenic diet and physical activity have the potential to reverse some of these

14 obesity-associated alterations; however, more clinical data is needed to confirm

15 translation to human subjects.

\section{Abstract}

18 The prevalence of obesity, an established risk factor for many cancers, has increased

19 dramatically over the past 50 years in the United States and many other countries.

20 Relative to normoweight cancer patients, obese cancer patients often have poorer

21 prognoses, resistance to chemotherapies, and are more likely to develop distant

22 metastases. Recent progress on elucidating the mechanisms underlying the obesity-cancer

23 connection suggests that obesity exerts pleomorphic effects on pathways related to tumor 
24 development and progression, and thus there are multiple opportunities for prevention

25 and treatment of obesity-related cancers. We now know that obesity can impact each of

26 the well-established hallmarks of cancer, but obesity-associated perturbations in systemic

27 metabolism and inflammation, and the interactions of these perturbations with cancer cell

28 energetics, are emerging as the primary drivers of obesity-associated cancer development

29 and progression. Several obesity-related host factors, including components of the

30 adipose secretome and structural components of the tumor microenvironment, are

31 extrinsic to, and interact with, the intrinsic molecular characteristics of cancer cells

32 (including cancer stem cells). Each will be considered in the context of potential

33 preventive and therapeutic strategies to reduce the burden of obesity-related cancers.

34 This review will focus on current knowledge of the mechanisms behind the obesity-

35 cancer link as well as relevant dietary and lifestyle interventions that are being

36 implemented in preclinical and clinical trials, with the ultimate goal of reducing

37 incidence and progression of obesity-related cancers.

39 Abbreviations : AMP kinase (AMPK); body mass index (BMI); brown adipose tissue

40 (BAT); calorie restriction (CR); cardiovascular disease (CVD); estrogen receptor (ER);

41 free fatty acids (FFA); insulin-like growth factor-1 (IGF-1); interleukin (IL); intermittent

42 fasting (IF); ketogenic diet (KD); monocyte chemo-attractant protein-1 (MCP-1);

43 mammalian target of rapamycin (mTOR); non-alcoholic steatohepatitis (NASH); nuclear

44 factor kappa-light-chain-enhancer of $\mathrm{B}$ cells $(\mathrm{NF} \mathrm{kB})$; plasminogen activator inhibitor-1

45 (PAI-1); phospatidylinositol-3 kinase (PI3K); peroxisome proliferator-activated receptor

46 (PPAR); physical activity (PA); signal transducer and activator of transcription (STAT); 
47 tumor necrosis factor- $\alpha$ (TNF- $\alpha$ ); type II Diabetes (T2DM); vascular endothelial growth 48 factor (VEGF); white adipose tissue (WAT)

49 


\section{Introduction}

51 Over the past half century in the United States the prevalence of obesity, defined as body

52 mass index (BMI) of $30 \mathrm{~kg} / \mathrm{m}^{2}$ or greater, has tripled. Today nearly $40 \%$ of adults and

$5320 \%$ of children in the United States are obese ${ }^{1}$. Worldwide, more than 600 million adults

54 are obese and 2.1 billion are overweight ${ }^{2}$. Obesity increases risk of several chronic

55 diseases and comorbidities including type II diabetes (T2DM), cardiovascular disease

56 (CVD), hypertension, chronic inflammation and, as discussed in this review, many types

57 of cancer ${ }^{3}$.

58

59 As illustrated in Figure 1, and based on the recent report from the International Agency

60 for Research on Cancer, risk of 13 distinct cancer types is increased with excess body

61 fatness ${ }^{4}$. These obesity-associated cancers include breast (in postmenopausal women),

62 ovarian, liver, gallbladder, kidney, colon, pancreatic, gastric, esophageal, endometrial,

63 thyroid, multiple myeloma, and meningioma ${ }^{4}$. Overall, an estimated $13 \%$ of incident

64 cases worldwide, and approximately $20 \%$ of incident cases in Europe and North America,

65 are attributable to obesity ${ }^{5}$. Aside from higher risk of developing cancer, obese

66 individuals are more likely to have reduced response to anticancer therapies ${ }^{6}$, and obesity

67 is implicated in approximately $20 \%$ of all cancer-related mortalities ${ }^{7}$. This includes

68 prostate cancer, for which obesity increases progression but not incidence ${ }^{8}$. Here, we

69 discuss (with a focus on developing mechanism-based intervention strategies) many ways

70 in which obesity can influence normal epithelial tissue homeostasis and cancer

71 development and/or progression, including metabolic perturbations involving hormonal, 
72 growth factor and inflammatory alterations, as well as interactions with the

73 microenvironment.

74

\section{Methods}

76 A traditional literature review was performed to describe the multiple mechanisms

77 underlying the obesity-cancer link, as well as dietary interventions targeting those

78 mechanisms for cancer prevention and treatment. Searches were completed using

79 PubMed and Google Scholar. A variety of key words were searched including obesity,

80 metabolic syndrome, cancer prevention, cancer treatment, calorie restriction, intermittent

81 fasting, ketogenic diet, and physical activity.

\section{Obesity Impacts Each Hallmark of Cancer}

84 Hanahan and Weinberg identified essential biological capabilities acquired by all cancer

85 cells during the multistep development of tumors in their classic article titled "The

86 Hallmarks of Cancer" first published in $2000^{9}$ and updated in their 2011 "Hallmarks of

87 Cancer: the Next Generation"10. These essential aberrations of cancer cells, include

88 sustaining proliferative signaling, increased chronic inflammation, evading growth

89 suppressors, resisting cell death, displaying genome instability, enabling replicative

90 immortality, inducing angiogenesis, and activating processes related to invasion and

91 metastasis. Conceptual progress in the decade between these two articles led to

92 identification of additional hallmarks, including reprogramming of energy metabolism,

93 evading immune destruction, and creation of the tumor microenvironment through

94 recruitment of various non-cancerous cells. Emerging evidence supports the concept that 
95 metabolic reprogramming, inflammation, and genome instability (including epigenetic

96 changes) represent the "hallmarks of hallmarks" and underlie many of the other essential

97 aberrations of cancer. In the case of cancer-associated metabolic reprogramming, cancer

98 cells often preferentially metabolize glucose through glycolysis rather than oxidative

99 phosphorylation (even under normoxic conditions) to generate substrate for cell

100 division $^{10-12}$. Thus, citric acid cycle intermediates not utilized for ATP production are

101 shuttled out of the mitochondria providing precursors for nucleotide, amino acid and lipid

102 synthesis pathways for the dividing cell ${ }^{12}$. In this way, cancer cells readily take up and

103 metabolize glucose to provide substrate for daughter cell production, with glucose

104 transporters and glycolytic enzymes being elevated in most cancers ${ }^{13}$.

106 Metabolic Syndrome and Systemic Metabolic Perturbations

107 Interactions between cellular energetics in cancer cells and systemic metabolic changes

108 associated with obesity are emerging as critical drivers of obesity-related cancer.

109 Intrinsically linked with obesity and associated with alterations in several cancer-related

110 host factors is metabolic syndrome, characterized by insulin resistance, hyperglycemia,

111 hypertension and dyslipidemia. In both obesity and metabolic syndrome, alterations occur

112 in circulating levels of insulin and insulin-like growth factor (IGF)-1; adipokines (e.g.

113 leptin, adiponectin, resistin, and monocyte chemotactic protein (MCP)-1); inflammatory

114 factors (e.g. interleukins (IL)-6, 10, and 17, interferon- $\gamma$ and tumor necrosis factor (TNF)-

$115 \alpha$ ); several chemokines; lipid mediators such as prostaglandin E2; and vascular-

116 associated factors (e.g. vascular endothelial growth factor (VEGF) and plasminogen

117 activator inhibitor (PAI)-1 $)^{14-16}$. Each of these factors has a putative role in development 
118 and progression of cancer, as well as a number of other chronic diseases ${ }^{14,16}$ including

119 CVD and T2DM, and will be explored in more detail below.

\section{Insulin, IGF-1 and Growth Factor Signaling}

122 In response to elevated blood glucose level, pancreatic $\beta$-cells release insulin, a peptide

123 hormone that stimulates peripheral uptake of glucose, glucose metabolism, and energy

124 storage pathways. As depicted in Figure 2, obesity and metabolic syndrome are

125 characterized by hyperglycemia and associated aberrations in insulin signaling, growth

126 factor signaling, and glucose metabolism ${ }^{17}$. One growth factor implicated in cancer risk

127 and progression is IGF-1. Produced primarily following growth hormone stimulation in

128 the liver, IGF-1 functions as a regulator of growth and development processes ${ }^{18}$. IGF

129 binding proteins bind to IGF-1 in circulation and limit its bioavailability to bind to IGF-1

130 receptor and induce downstream signaling to promote growth and/or survival ${ }^{19}$.

131 Hyperglycemia and hyperinsulinemia, hallmarks of metabolic syndrome, increase IGF-1

132 production and bioavailability. Hyperglycemia suppresses IGF-1 binding protein

133 synthesis and hyperinsulinemia promotes expression of growth hormone receptor and

134 subsequent IGF-1 synthesis ${ }^{17}$. Growth and survival functions of IGF-1 give it the

135 potential to impact many hallmarks of cancer, including suppression of apoptosis and

136 promotion of cell cycle progression, angiogenesis and metastatic potential ${ }^{20}$. As a result,

137 elevated IGF-1 is established as a risk factor for many types of cancer ${ }^{19}$.

139 IGF-1 receptor and insulin receptor stimulate the same downstream activation of

140 phosphoinositide 3-kinase (PI3K)/Akt pathway (Figure 2), a pathway frequently altered 
141 in epithelial cancers ${ }^{21}$. In response to these growth factors and nutrient availability,

$142 \mathrm{PI} 3 \mathrm{~K} /$ Akt produces lipid messengers that initiate the Akt signaling cascade ${ }^{21}$, activating

143 downstream mammalian target of rapamycin (mTOR) ${ }^{22}$. When activated mTOR initiates

144 downstream signaling that promotes cell growth, proliferation and survival. In response

145 to low nutrient conditions AMP-activated kinase (AMPK), another energy responsive

146 pathway, inhibits mTOR activation and downstream signaling ${ }^{23}$. Oncogenic signals or

147 loss of tumor suppressors can activate mTOR and contribute to the hallmarks of cancer,

148 promoting proliferation, survival, angiogenesis, and metastasis ${ }^{24}$. In preclinical models,

149 blocking mTOR signaling with drugs such as rapamycin (mTOR inhibitor) ${ }^{25-27}$ and

150 metformin (AMPK activator) ${ }^{25,28,29}$, block tumor-enhancing effects associated with the

151 obese phenotype ${ }^{30}$. Interestingly, rapamycin has exhibited anti-inflammatory attributes,

152 attenuating inflammation as well as tumor promotion, suggesting crosstalk between

153 mTOR-related growth and survival signals and inflammatory signals ${ }^{31}$.

154

\section{Chronic Inflammation: The Role of Adipose Tissue}

156 Mammals, including humans, have 2 major fat depots: subcutaneous and visceral (intra-

157 abdominal). These adipose depots contain white adipose tissue (WAT) that stores energy

158 in the form of triacylglycerol and brown adipose tissue (BAT) that dissipates energy by

159 burning fatty acids to generate heat. WAT and BAT have important differences in their

160 morphology, metabolism and transcriptional profiles. White adipocytes have few

161 mitochondria, low oxidative rate, and contain a unilocular lipid droplet comprised

162 primarily of triacylglycerol, while brown adipocytes have a high number of mitochondria

163 (hence the darker appearance), high rate of fatty acid and glucose uptake and oxidation, 
164 and possess multilocular lipid droplets $^{32}$. Moreover, the secretome of white versus brown 165 adipocytes differs markedly (Figure 3); the former is characterized by secretion of leptin, 166 resistin, PAI-1, inflammatory cytokines, and free fatty acids (FFA), while the latter is

167 characterized by secretion of bone morphogenetic proteins, lactate (which induces

168 uncoupling proteins), retinaldehyde, triiodothyonine (T3) and other factors associated

169 with response to cold stress and/or increased energy expenditure ${ }^{32}$. Brown adipocytes

170 also produce adiponectin (but not leptin) and fibroblast growth factor-21, which can be

171 anti-inflammatory and insulin sensitizing ${ }^{32}$. WAT also contains a number of stromal cells

172 including pre-adipocytes, vascular cells, fibroblasts and a host of immune cells such as

173 adipose tissue macrophages ${ }^{33}$. Increased WAT mass in obesity drives chronic

174 inflammation in at least 3 ways, depicted in Figure 4 and summarized below:

175

176 1. Altered Adipose Secretome

177 Leptin is an energy-sensing peptide hormone produced by adipocytes. Leptin levels,

178 positively correlated with adiposity, function as an energy sensor through signaling to the

179 hypothalamus, decreasing hunger cues, food intake and weight gain. Leptin release from

180 adipocytes is stimulated by a variety of factors including insulin, TNF $\alpha$, glucocorticoids,

181 and estrogen ${ }^{34}$. In obesity, leptin is overproduced by adipocytes, reducing hypothalamic

182 sensitivity to the signal ${ }^{35}$. Circulating leptin binds to various receptors in central nervous

183 system and peripheral tissues, regulating processes including energy homeostasis,

184 cytokine production, immune function, and carcinogenesis ${ }^{34,36}$. The leptin receptor OB-R,

185 classified as a class I cytokine receptor, gives leptin the ability to activate signal

186 transducer and activator of transcription (STAT) family transcription factors, resulting in 
187 initiation of STAT-induced transcription programs for proliferation, cell growth and

188 survival, migration and differentiation ${ }^{37}$. Deregulation of STATs activity is often

189 observed in cancer ${ }^{38}$.

191 Adiponectin, another peptide hormone secreted from adipocytes, functions as an energy

192 sensor that promotes hunger and energy intake, opposing the functions of leptin.

193 Although the most abundant hormone secreted from the WAT, adiponectin levels are

194 negatively correlated with adiposity and release is stimulated during energy deficit.

195 Adiponectin opposes obesity-associated metabolic alterations through regulating glucose

196 metabolism, increasing insulin sensitivity and fatty acid oxidation, and reducing IGF-1

197 signaling through activation of AMPK, inhibitor of downstream mTOR ${ }^{39}$. Adiponectin

198 also attenuates inflammation through inhibition of nuclear factor kappa-light-chain-

199 enhancer of B cells (NF- $\mathrm{B}$ ), which reduces expression of proinflammatory cytokines

200 while increasing expression of anti-inflammatory cytokines ${ }^{40}$. Due to the anticancer

201 functions of adiponectin, adiponectin agonists are emerging as possible chemotherapeutic

202 agents, particularly for obesity-related cancers ${ }^{41}$. While associations between each of

203 these adipokines and cancer risk are established, the leptin to adiponectin ratio is

204 increasingly considered a more sensitive measure in evaluating cancer risk ${ }^{42}$.

206 Sex hormones, including estrogen, androgens and progestogens, regulate a variety of

207 growth and developmental processes including weight homeostasis ${ }^{43}$. Long established is

208 the association between sex hormone levels and obesity ${ }^{44}$. In postmenopausal women,

209 BMI is positively correlated with estrone, estradiol, and free estradiol ${ }^{45}$. Elevation of 
210 estrogens is also detected in obese men $^{44,46}$; however, testosterone levels are significantly

211 reduced $^{47}$. Alteration of sex hormones can result in several biological disorders including

212 hypertension, menstrual disturbances, erectile dysfunction, gynecomastia, hirsutism, and

213 increased adiposity ${ }^{44}$. Moreover, sex hormones have been implicated in risk and/or

214 progression of multiple cancer types ${ }^{48}$. In prostate cancer, sex hormone levels are

215 associated with disease progression, not disease risk ${ }^{49}$. Low levels of circulating

216 testosterone correlates with aggressive disease progression ${ }^{50}$. Elevated estrogen levels are

217 associated with increased risk of breast $\mathrm{t}^{44,45,51}$ ovarian $^{52}$, and endometrial cancers ${ }^{53}$.

219 Menopausal status can also modulate sex hormone secretion and signaling in women.

220 Prior to menopause, ovaries are the main site of estrogen production, whereas after

221 menopause, peripheral sites including adipose tissue, are the main source of estrogen

222 production. In postmenopausal, obese women adipose tissue serves as the main site of

223 estrogen synthesis ${ }^{45}$. Once released, circulating estrogens bind to one of two estrogen

224 receptors (ER), ER $\alpha$ or ER $\beta$. Once bound, receptors dimerize and translocate to the

225 nucleus where they bind to DNA or other transcription factors, influencing gene

226 expression profiles that regulate growth, proliferation and differentiation ${ }^{54}$. In the context

227 of cancer, the two receptors have differing roles. ER $\alpha$ is mitogenic and an established

228 target in treatment of estrogen receptor-positive breast cancer, while ER $\beta$ is suggested to

229 be tumor suppressive ${ }^{55}$. Obesity and postmenopausal status increases risk of ER-positive

230 breast cancers compared with ER-negative breast cancer ${ }^{56}$. Due to the positive

231 association between obesity, circulating estrogen and risk of ER-positive breast cancer, 
aromatase inhibitors and ER antagonist, including tamoxifen, have been investigated for

233 their effectiveness as adjuvant therapy ${ }^{57}$.

\section{2. Crown-Like Structures}

236 Obesity drives subclinical inflammation in visceral and subcutaneous WAT,

237 characterized by crown-like structures, or rings of activated macrophages surrounding

238 engorged or necrotic adipocytes (Figure 4). This adipocyte-macrophage interaction

239 results in a proinflammatory secretome from both cell types, activating the cellular

240 transcription factor NF-kB, increasing levels of cytokines and other inflammatory factors,

241 and triggering inflammation ${ }^{58}$.

\section{3. Adipose Remodeling and Lipid Infiltration in Other Tissues}

244 During conditions of low nutrient availability or increased energy needs, glucagon

245 secretion stimulates lipolysis of adipocytes, releasing FFA into the blood stream ${ }^{59}$.

246 Circulating FFA can then be utilized by peripheral tissues, providing substrate for $\beta$ -

247 oxidation and serving as intermediates for energy production through the citric acid cycle

248 and oxidative phosphorylation. Overnutrition remodels existing adipose tissue, expanding

249 adipocyte number and size, and altering adipokine secretion, FFA flux, and adipocyte

250 death $^{60}$. In response, adipose stromal cells modify their functions to promote clearance of

251 necrotic adipocytes and generation of new adipocytes and vasculature. Tissue remodeling

252 in chronic overnutrition or obesity, results in sustained, low-grade inflammation and

253 metabolic alterations ${ }^{60}$. As stated above, cancer cells adapt to changing energy needs for

254 proliferation through metabolic reprogramming, increasing anaerobic metabolism and 
255 shunting citric acid cycle intermediates to synthetic pathways ${ }^{10,12}$. Production of daughter 256 cells demands increased levels of FFA for formation of lipid bilayers, thus excess WAT 257 promotes proliferation of tumor cells through provision of circulating FFA $^{61}$.

259 Chronic overnutrition can lead to lipid accumulation beyond capacity of adipose depots,

260 leading to deposition of lipids in peripheral tissues including muscle, liver and pancreatic

261 tissue $^{62}$. Ectopic lipid intermediates exert lipotoxic effects, impairing cellular organelle

262 functions, releasing inflammatory cytokines, and fostering development of insulin

263 resistance ${ }^{63}$. Consequently, individuals can develop muscle dysfunction and hepatic and

264 pancreatic steatosis, all of which have been positively correlated with insulin resistance

265 and impaired lipid metabolism ${ }^{62}$.

267 Nonalcoholic fatty liver disease, diagnosed as $>5-10 \%$ liver fat content by weight in the

268 absence of alcohol use or other liver disease, encompasses a variety of liver diseases

269 including simple steatosis, nonalcoholic steatohepatitis (NASH) and cirrhosis ${ }^{64}$. One of

270 the most common chronic diseases ${ }^{65-67}$, Nonalcoholic fatty liver disease is present in 65-

$27185 \%$ of obese patients ${ }^{64,68}$ with rapidly rising incidence among adults and children ${ }^{66,69}$.

272 Excess accumulation of lipids in the liver, exerts lipotoxic effects including production of

273 reactive oxygen species, activation of pro-inflammatory programs, and endoplasmic

274 reticular stress, impairing function of cellular organelles and potentially inducing hepatic

275 cell death ${ }^{70}$. Additionally, accumulation of lipids and pro-inflammatory cytokines

276 promotes activation of intracellular kinases, leading to impaired insulin signaling and

277 development of insulin resistance ${ }^{71}$. While simple steatosis is benign, NASH is more 
278 detrimental, characterized by liver injury, inflammation and/or fibrosis. NASH can

279 further result in the development of cirrhosis, liver failure, and hepatocellular

280 carcinoma $^{72}$

281

282 Deposition of adipocytes in the pancreas appears to occur early in obesity-associated

283 pancreatic dysfunction, altering secretion and signaling of endocrine factors including

284 insulin. Infiltrating fat in the pancreas has been associated with increased visceral WAT

285 mass and insulin resistance $\mathrm{e}^{73,74}$. These endocrine alterations further complicate the

286 complex metabolic and inflammatory perturbations characterized in obesity and

287 metabolic syndrome and can trigger the development of pancreatic steatosis, pancreatitis

288 and/or nonalcoholic fatty pancreatic disease, established risk factors for pancreatic

289 cancer $^{73,74}$.

291 Angiogenesis

292 As adipose tissue depots expand in obesity, the existing vasculature must expand to meet

293 demand. This outgrowth of new blood vessels is termed angiogenesis. Key mediators of

294 this process include VEGF and PAI-1. VEGF, is a potent angiogenic factor that is

295 produced by adipocytes and tumor cells. VEGF acts on endothelial cells stimulating

296 mitogenic and vascular permeability-enhancing activities ${ }^{75}$. Obesity is associated with

297 increased circulating VEGF, and elevated VEGF correlates with poor prognosis for many

298 obesity-related cancers $^{76}$. PAI-1 is another angiogenic factor, produced by adipocytes,

299 endothelial cells, and stromal cells in visceral $\mathrm{WAT}^{77}$, that is frequently elevated in obese

300 subjects. Increased circulating PAI-1 is associated with increased risk of other chronic 
301 diseases including CVD, T2DM and a number of cancers ${ }^{77}$. While interaction of

302 angiogenic factors with proximal endothelial cells induce formation of local blood

303 vessels, providing a route for oxygen and nutrient delivery and waste removal, these

304 factors can also interact with peripheral tissues, facilitating angiogenesis, and potentially

305 promoting progression at tumor sites. These newly formed blood vessels would

306 potentially provide primary tumor mass with oxygen and nutrients to sustain proliferation

307 and survival as well as a route for metastasis to distant sites. PAI-1 functionally inhibits

308 plasminogen activators, thus regulating extracellular matrix integrity ${ }^{78}$. Extracellular

309 matrix remodeling is a key feature of invasive disease, and integral in the development of

310 metastatic lesions ${ }^{79}$. Due to the antitumorigenic potential of factors that modulate

311 angiogenesis, targeted drugs have been developed. However, caution should be advised

312 in administration of anti-angiogenic treatments in obese patients, as these drugs can

313 induce hypoxia in primary tumors, potentially encouraging metastasis, already a concern

314 in the obese population ${ }^{79}$. Elevation of these factors may also impact efficacy of

315 treatment regimens, as excess circulating VEGF in obese patients contributes to reduced

316 efficacy of anti-VEGF therapies (e.g. bevacizumab) compared with non-obese ovarian

317 cancer patients ${ }^{80}$.

319 Dietary Interventions Targeting Obesity for Cancer Prevention and Treatment

320 Given the multifaceted role of obesity in promoting a protumorigenic microenvironment

321 that facilitates tumor development and progression, interventions are urgently needed to

322 break the obesity-cancer link. To date, the only weight loss intervention in obese people

323 consistently associated with reduced cancer risk is bariatric surgery ${ }^{81}$. In light of the 
324 expense and complications inherent in surgical weight loss approaches, current efforts are

325 focusing on reducing adiposity through lifestyle and dietary interventions. To achieve

326 reductions in weight and adiposity these interventions have aimed to 1) promote negative

327 energy balance through either reduced energy intake via calorie restriction (CR) or

328 intermittent fasting (IF) or through increased energy expenditure via physical activity

329 (PA) or 2) implementation of ketogenic diet (KD) a dietary pattern associated with

330 weight loss and reduced cancer progression. Preclinical and some clinical studies suggest

331 that these interventions can favorably and inversely modulate cancer risk biomarkers

332 including insulin, IGF-1, leptin, adiponectin, cytokines, angiogenic factors, and crown-

333 like structures compared to the obese state. Modulation of these biomarkers could result

334 in downstream reductions in growth factor signaling, inflammation, and angiogenesis,

335 attenuating cancer risk and progression (Figure 5).

337 1. Calorie Restriction

338 Calorie restriction (CR), defined as reduction of dietary energy intake without

339 malnutrition, is broadly effective dietary intervention that significantly decreases

340 adiposity. Preclinical models demonstrate 30\% CR, compared with ad libitum-fed

341 control, ameliorates risk factors and delays onset of cancer through metabolic alterations

342 fostering increased insulin sensitivity and decreased serum glucose, growth factor

343 signaling, inflammation, oxidative stress and angiogenesis ${ }^{82-85}$. These metabolic changes

344 translate into significantly decreased cancer incidence in murine models ${ }^{86}$. Due to long

345 latency of cancer in humans, the literature does not have data linking CR directly with

346 cancer incidence in humans. However, randomized control trials implementing long-term 
$34720 \%$ CR in overweight human subjects has confirmed reduced adiposity, improved

348 glucose homeostasis, increased adiponectin, and reduced leptin and inflammatory

349 markers TNF $\alpha$ and C-reactive protein ${ }^{87,88}$. Substantial weight loss of $>10 \%$ may be

350 necessary to consistently gain these benefits ${ }^{89-91}$.

352 Limited clinical studies exist on CR during cancer treatment. Direct application of CR in

353 cancer patients is complicated by high rates of weight loss associated with cancer

354 cachexia, a condition in which tumor-derived signals degrade muscle and adipose tissue.

355 Emerging findings from preliminary clinical trials suggest that application of CR as an

356 adjuvant therapy in combination with chemotherapy and/or radiation has potential to

357 increase responsiveness to treatment ${ }^{92,93}$.

359 2. Intermittent Fasting

360 Preclinical and clinical studies have begun to explore implementation of intermittent

361 fasting (IF), which may be easier for most people to adopt and may have beneficial

362 metabolic effects relative to chronic CR. Human trials most often study one of three IF

363 regimens: alternate day fasting, alternate day energy restriction $(\sim 75 \%)$ or 2 consecutive

364 days of $65 \%$ energy restriction, the latter often referred to as intermittent calorie

365 restriction ${ }^{94}$. Periods of IF stimulate reduced insulin and increased glucagon, resulting in

366 increased lipolysis and fatty acid oxidation to provide alternate substrates for energy

367 production. These metabolic alterations are accompanied by reductions in several cancer-

368 related risk factors including lower insulin resistance, inflammation, and circulating IGF-

$3691^{95}$. The impact of IF on angiogenesis in the context of cancer remains unexplored in 
370 currently published research. Preclinical studies with IF consistently exhibit a cancer

371 preventative effect with reduced rates of tumor growth for multiple cancer types ${ }^{95-97}$. To

372 our knowledge there is no published data on IF and cancer incidence in human subjects,

373 although there are reports of favorable effects of IF in overweight human, including

374 improved adipokine ratios and reduced inflammation ${ }^{96,98}$, suggesting the reported

375 preclinical anticancer effects of IF may be translatable to humans.

377 One IF regimen being examined as a breast cancer prevention strategy is called the 5:2

378 diet and involves 5 days/week of a healthy diet, such as the Mediterranean diet, with two

379 consecutive days of a low calorie, low carbohydrate diet. The Mediterranean diet is

380 primarily a plant-based diet high in fruits, vegetables, whole grains, legumes and nuts.

381 Compared to North American dietary patterns, the Mediterranean diet has been

382 associated with better control of body weight, reduction of cancer risk biomarkers and

383 decreased cancer incidence ${ }^{99-103}$. The diet results in favorable modulation of

384 inflammation, oxidative stress, and growth factor signaling. Combining a Mediterranean

385 diet with 2 days of a very low calorie, low carbohydrate diet for one month in 24 obese

386 women at high risk for breast cancer induced changes in breast tissue gene expression

387 and metabolites associated with reduced risk of breast cancer ${ }^{104}$.

389 Regarding the effects of IF on cancer prognosis, a study by Safdie, et al suggests IF

390 during cancer therapy may decrease adverse effects of chemotherapy. Ten cancer patients

391 (various cancer types) voluntarily fasted prior to (48-140 hours) or following (5-56 hours)

392 chemotherapy treatment. Compared with non-restricted control subjects, fasting reduced 
chemotherapy-induced side effects including fatigue, weakness and gastrointestinal side

394 effects while exhibiting the same chemotherapy-induced reduction in tumor volume or

395 biomarkers ${ }^{105}$. Following this ground breaking study, others have implemented IF in

396 small scale clinical trials including de Groot, S., et al., 2015, where short term IF among

397 stage II/III breast cancer patients was well tolerated, reduced signs of hematological

398 toxicity and stimulated faster recovery from DNA damage in normal host peripheral

399 blood mononuclear cells ${ }^{106}$. Limited preclinical findings suggest that IF may selectively

400 protect healthy cells and make cancer cells more vulnerable to chemotherapeutic agents,

401 reducing side-effects and increasing drug efficacy ${ }^{95}$. More research is needed to confirm

402 these findings and identify underlying mechanisms.

405 3. Physical Activity

406 Engaging in physical activity (PA), alone or in combination with reduced dietary energy

407 intake, can be another effective method in generating a negative energy balance, reducing

408 weight and adiposity. A published systematic review of the literature on PA in cancer

409 survivors revealed that PA produced favorable modulation of insulin/IGF-1 pathways and

410 inflammation ${ }^{107}$. Limited evidence from preclinical studies suggest that PA may also

411 reduce the level of intratumoral mTOR activation, VEGF expression and

412 angiogenesis ${ }^{108,109}$. Intervention studies suggest that reduction in these risk biomarkers

413 associated with PA may be reliant on significant weight loss ${ }^{110-112}$. Furthermore, the

414 amount of exercise can influence effectiveness of PA. For example, in one study PA did

415 not significantly reduce inflammatory markers unless participants achieved 120 minutes 
416 per week, just short of the American Cancer Society's recommendation of 150

417 minutes $^{113}$. Epidemiological and cohort studies confirm an anticancer potential and

418 demonstrate a 20-30\% reduction in cancer risk with substantial PA for multiple cancer

419 types including breast, colon and endometrial ${ }^{114}$.

420

421 PA is also safe and beneficial during cancer therapy for multiple cancer types ${ }^{115-117}$. Not

422 only can PA improve body composition, it can also reduce unwanted side effects of

423 treatment and improve physical functioning and quality of life parameters. A randomized

424 control trial in stage II breast cancer patients found that 10 weeks of interval-based,

425 aerobic exercise reduced chemotherapy-induced nausea and increased individual

426 functional capacity ${ }^{118,119}$. Courneya, et al. findings suggest that PA may increase

427 chemotherapy completion rate without causing adverse events such as lymphedema in

428 breast cancer patients ${ }^{120}$. Benefits are further exhibited in elderly patients with exercise

429 during treatment improving memory and self-reported health and reducing fatigue ${ }^{121}$.

430 Studies on exercise during treatment suggest that higher-intensity exercise provides more

431 benefit than low-intensity exercise ${ }^{122}$.

432

433 Despite the observed positive benefits of PA, important questions remain regarding

434 intensity and amount of physical activity that must be performed to fully reap the

435 benefits. Based on current knowledge, the American Cancer Society advises 150 minutes

436 of moderate or 75 minutes of vigorous per week for cancer prevention and

437 survivorship ${ }^{123}$. 
440 Ketogenic diet (KD) is a very-low carbohydrate diet with high fat and moderate protein

441 composition. Low carbohydrate consumption reduces available glucose, a cancer cell's

442 preferred energy source, and increases catabolism of proteins and fats to provide

443 gluconeogenic glucose and ketones. With prolonged consumption of KD, glycogen stores

444 reach critical levels and the body is no longer able to oxidize fats to glucose via

445 gluconeogenesis. This results in a shift to increased ketone production and physiological

446 ketosis. Ketosis is not to be confused with ketoacidosis that is seen with diabetes mellitus.

447 In ketosis there is less accumulation of ketones, as they are being used efficiently by the

448 brain and body as an energy source, and individuals do not experience adverse side

449 effects associated with ketoacidosis ${ }^{124}$. Ketosis from KD favorably modulates many

450 cancer risk biomarkers including IGF-1, leptin, adiponectin, inflammatory markers, and

451 angiogenic factors (Figure 5) ${ }^{125-128}$. Preclinical studies suggest that KD can attenuate

452 these markers without a reduction in caloric intake; however, weight loss may be

453 needed ${ }^{129,130}$. KD may induce weight loss via several interrelated mechanisms, including:

454 reduced appetite due to high protein intake, which can induce higher satiety, and high

455 ketones, known to modulate appetite-regulating hormones; reduced caloric intake due to

456 the satiety; reduced lipogenesis and increased lipolysis; greater metabolic efficiency; and

457 increased metabolic cost of gluconeogenesis and ketogenesis ${ }^{124}$.

459 Beneficial effects of the ketogenic diet have long been established for epilepsy and

460 T2DM; emerging is its role in cancer prevention and treatment ${ }^{124}$. Early preclinical

461 studies found KD reduced tumor burden and cachexia in a mouse model of colon 
462 cancer $^{131}$. Further preclinical models have confirmed these findings and extended benefits

463 of decreased tumor growth and increased survival to other cancer types including

464 malignant glioma, gastric and prostate cancers ${ }^{132}$. To date results from clinical trials

465 focused on implementation of KD in cancer prevention and treatment have been limited,

466 and ongoing clinical trials are addressing this gap in the literature with multiple cancer

467 types $^{133}$.

469 It is important to also consider potential adverse effects of KD. Reduction of

470 carbohydrate in KD is replaced with increased protein and fat. High protein intake has

471 been linked to kidney damage ${ }^{134}$, although this is not widely accepted with other

472 preclinical, human, and meta-analysis studies finding no evidence of renal damage with

473 high protein intake ${ }^{124}$. Additionally, select preclinical studies have found long-term KD

474 to cause dyslipidemia, hepatic steatosis and glucose intolerance ${ }^{135}$. More research is

475 needed to evaluate the safety and efficacy of ketogenic diets as cancer prevention and

476 treatment interventions.

477

478 Summary and Conclusions

479 A strong link between obesity and cancer risk has been established in the epidemiological

480 and preclinical literature. Obesity is associated with several systemic metabolic

481 perturbations that are correlated with increased cancer risk and/or poor prognosis,

482 including dysregulation of insulin and growth factor signaling, adipokine signaling,

483 inflammation, and angiogenesis. Establishment of this obesity-cancer link has spurred

484 research focused on a variety of lifestyle and dietary interventions to promote a negative 
energy balance, attain weight loss, attenuate risk biomarkers, and prevent obesityassociated cancers. Preclinical and early clinical work on these putative anticancer dietary

487 and lifestyle interventions, including CR, PA, IF, and KD, are also being evaluated, some

488 showing promise in reducing cancer risk. Additionally, the literature suggests that these

489 interventions may improve response to chemotherapy for multiple cancer types. While

490 many clinical studies have evaluated the safety and efficacy of PA as adjuvant therapy

491 and suggest it is safe for patients, there are few clinical trials that evaluate the utilization

492 of dietary interventions such as $\mathrm{CR}, \mathrm{IF}$, and $\mathrm{KD}$ as adjuvant therapy (Table 1). Future

493 studies will need to focus on the safety and added benefit to current therapies, and should

494 also consider the potential of the dietary interventions to sensitize patients and facilitate

495 the use of lower doses of chemotherapy or radiation therapy to improve therapeutic

496 response.

\section{References}

499 1. Flegal KM, Carroll MD, Kit BK, Ogden CL. Prevalence of obesity and trends in 500 the distribution of body mass index among US adults, 1999-2010. JAMA. $501 \quad$ 2012;307(5):491-497.

502 2. Ng M, Fleming T, Robinson M, et al. Global, regional, and national prevalence 503 of overweight and obesity in children and adults during 1980-2013: a 504 systematic analysis for the Global Burden of Disease Study 2013. Lancet. $505 \quad$ 2014;384(9945):766-781.

506 3. Khaodhiar L, McCowen KC, Blackburn GL. Obesity and its comorbid conditions. 507 Clinical cornerstone. 1999;2(3):17-31. 
508 4. Lauby-Secretan B, Scoccianti C, Loomis D, et al. Body Fatness and Cancer-509 Viewpoint of the IARC Working Group. N Engl J Med. 2016;375(8):794-798.

510 5. Arnold M, Pandeya N, Byrnes G, et al. Global burden of cancer attributable to 511 high body-mass index in 2012: a population-based study. The Lancet Oncology. $512 \quad 2014$.

513 6. Lashinger LM, Rossi EL, Hursting SD. Obesity and resistance to cancer 514 chemotherapy: interacting roles of inflammation and metabolic dysregulation. 515 Clinical pharmacology and therapeutics. 2014;96(4):458-463.

516 7. Calle EE, Rodriguez C, Walker-Thurmond K, Thun MJ. Overweight, obesity, and 517 mortality from cancer in a prospectively studied cohort of U.S. adults. $N$ Engl J $518 \quad$ Med. 2003;348(17):1625-1638.

519 8. Allott EH, Masko EM, Freedland SJ. Obesity and prostate cancer: weighing the 520 evidence. European urology. 2013;63(5):800-809.

521 9. Hanahan D, Weinberg RA. The hallmarks of cancer. Cell. 2000;100(1):57-70.

522 10. Hanahan D, Weinberg RA. Hallmarks of cancer: the next generation. Cell. $523 \quad 2011 ; 144(5): 646-674$.

524 11. Chen X, Qian Y, Wu S. The Warburg effect: evolving interpretations of an $525 \quad$ established concept. Free Radic Biol Med. 2015;79:253-263.

526 12. Ward PS, Thompson CB. Metabolic reprogramming: a cancer hallmark even 527 warburg did not anticipate. Cancer cell. 2012;21(3):297-308.

528 13. Ganapathy-Kanniappan S, Geschwind JF. Tumor glycolysis as a target for 529 cancer therapy: progress and prospects. Mol Cancer. 2013;12:152. 
530 14. Hursting SD, Berger NA. Energy balance, host-related factors, and cancer 531 progression. J Clin Oncol. 2010;28(26):4058-4065.

532 15. Hursting SD, Smith SM, Lashinger LM, Harvey AE, Perkins SN. Calories and 533 carcinogenesis: lessons learned from 30 years of calorie restriction research. $534 \quad$ Carcinogenesis. 2010;31(1):83-89.

535 16. Bonomini F, Rodella LF, Rezzani R. Metabolic syndrome, aging and 536 involvement of oxidative stress. Aging and disease. 2015;6(2):109-120.

537 17. Braun S, Bitton-Worms K, LeRoith D. The link between the metabolic 538 syndrome and cancer. International journal of biological sciences. $539 \quad$ 2011;7(7):1003-1015.

540 18. Agrogiannis GD, Sifakis S, Patsouris ES, Konstantinidou AE. Insulin-like growth 541 factors in embryonic and fetal growth and skeletal development (Review). $542 \quad$ Molecular medicine reports. 2014;10(2):579-584.

543 19. Pollak M. The insulin and insulin-like growth factor receptor family in 544 neoplasia: an update. Nat Rev Cancer. 2012;12(3):159-169.

545 20. Brahmkhatri VP, Prasanna C, Atreya HS. Insulin-like growth factor system in 546 cancer: novel targeted therapies. BioMed research international. $547 \quad 2015 ; 2015: 538019$.

548 21. Wong KK, Engelman JA, Cantley LC. Targeting the PI3K signaling pathway in 549 cancer. Current opinion in genetics \& development. 2010;20(1):87-90.

550 22. Memmott RM, Dennis PA. Akt-dependent and -independent mechanisms of 551 mTOR regulation in cancer. Cellular signalling. 2009;21(5):656-664. 
552 23. Hardie DG, Ross FA, Hawley SA. AMPK: a nutrient and energy sensor that 553 maintains energy homeostasis. Nature reviews Molecular cell biology. $554 \quad 2012 ; 13(4): 251-262$.

555 24. Populo H, Lopes JM, Soares P. The mTOR signalling pathway in human cancer. 556 International journal of molecular sciences. 2012;13(2):1886-1918.

557 25. Cifarelli V, Lashinger LM, Devlin KL, et al. Metformin and Rapamycin Reduce 558 Pancreatic Cancer Growth in Obese Prediabetic Mice by Distinct MicroRNA$559 \quad$ Regulated Mechanisms. Diabetes. 2015.

560 26. Athar M, Kopelovich L. Rapamycin and mTORC1 inhibition in the mouse: skin 561 cancer prevention. Cancer Prev Res (Phila). 2011;4(7):957-961.

562 27. Nogueira LM, Dunlap SM, Ford NA, Hursting SD. Calorie restriction and 563 rapamycin inhibit MMTV-Wnt-1 mammary tumor growth in a mouse model of 564 postmenopausal obesity. Endocr Relat Cancer. 2012;19(1):57-68.

565 28. Tomimoto A, Endo H, Sugiyama M, et al. Metformin suppresses intestinal polyp 566 growth in ApcMin/+ mice. Cancer science. 2008;99(11):2136-2141.

567 29. Chaudhary SC, Kurundkar D, Elmets CA, Kopelovich L, Athar M. Metformin, an 568 antidiabetic agent reduces growth of cutaneous squamous cell carcinoma by 569 targeting mTOR signaling pathway. Photochemistry and photobiology. $570 \quad 2012 ; 88(5): 1149-1156$.

571 30. De Angel RE, Conti CJ, Wheatley KE, et al. The enhancing effects of obesity on 572 mammary tumor growth and Akt/mTOR pathway activation persist after 573 weight loss and are reversed by RAD001. Mol Carcinog. 2013;52(6):446-458. 
574 31. Checkley LA, Rho O, Moore T, Hursting S, DiGiovanni J. Rapamycin is a potent 575 inhibitor of skin tumor promotion by 12-0-tetradecanoylphorbol-13-acetate. 576 Cancer Prev Res (Phila). 2011;4(7):1011-1020.

577 32. Saely $\mathrm{CH}$, Geiger K, Drexel H. Brown versus white adipose tissue: a mini578 review. Gerontology. 2012;58(1):15-23.

579 33. Eto H, Suga H, Matsumoto D, et al. Characterization of structure and cellular 580 components of aspirated and excised adipose tissue. Plastic and reconstructive surgery. 2009;124(4):1087-1097.

582 34. Gautron L, Elmquist JK. Sixteen years and counting: an update on leptin in energy balance. J Clin Invest. 2011;121(6):2087-2093.

584 35. Friedman JM, Mantzoros CS. 20 years of leptin: from the discovery of the leptin 585 gene to leptin in our therapeutic armamentarium. Metabolism. 2015;64(1):14.

587 36. Park HK, Ahima RS. Leptin signaling. F1000Prime Rep. 2014;6:73.

588 37. Mullen M, Gonzalez-Perez RR. Leptin-Induced JAK/STAT Signaling and Cancer $589 \quad$ Growth. Vaccines (Basel). 2016;4(3).

590 38. Yu H, Lee H, Herrmann A, Buettner R, Jove R. Revisiting STAT3 signalling in 591 cancer: new and unexpected biological functions. Nat Rev Cancer. 592 2014;14(11):736-746.

593 39. Vaiopoulos AG, Marinou K, Christodoulides C, Koutsilieris M. The role of 594 adiponectin in human vascular physiology. International journal of cardiology. 595 2012;155(2):188-193. 
596 40. Fantuzzi G. Adiponectin in inflammatory and immune-mediated diseases. 597 Cytokine. 2013;64(1):1-10.

598 41. Otvos L, Jr., Haspinger E, La Russa F, et al. Design and development of a 599 peptide-based adiponectin receptor agonist for cancer treatment. BMC $600 \quad$ biotechnology. 2011;11:90.

601 42. Ollberding NJ, Kim Y, Shvetsov YB, et al. Prediagnostic leptin, adiponectin, C602 reactive protein, and the risk of postmenopausal breast cancer. Cancer Prev Res (Phila). 2013;6(3):188-195.

604 43. Brown LM, Gent L, Davis K, Clegg DJ. Metabolic impact of sex hormones on obesity. Brain Res. 2010;1350:77-85.

606 44. Kirschner MA, Schneider G, Ertel NH, Worton E. Obesity, androgens, estrogens, 607 and cancer risk. Cancer Res. 1982;42(8 Suppl):3281s-3285s.

608 45. Cleary MP, Grossmann ME. Minireview: Obesity and breast cancer: the 609 estrogen connection. Endocrinology. 2009;150(6):2537-2542.

610 46. Meyer MR, Clegg DJ, Prossnitz ER, Barton M. Obesity, insulin resistance and diabetes: sex differences and role of oestrogen receptors. Acta Physiol (Oxf).

613 47. Allan CA, McLachlan RI. Androgens and obesity. Current opinion in 614 endocrinology, diabetes, and obesity. 2010;17(3):224-232.

615 48. Folkerd EJ, Dowsett M. Influence of sex hormones on cancer progression. J Clin Oncol. 2010;28(26):4038-4044.

617 49. Endogenous H, Prostate Cancer Collaborative G, Roddam AW, Allen NE, 618 Appleby P, Key TJ. Endogenous sex hormones and prostate cancer: a 
collaborative analysis of 18 prospective studies. J Natl Cancer Inst. 2008;100(3):170-183.

621 50. Schnoeller T, Jentzmik F, Rinnab L, et al. Circulating free testosterone is an 622 independent predictor of advanced disease in patients with clinically localized 623 prostate cancer. World J Urol. 2013;31(2):253-259.

624 51. Bernstein L, Ross RK. Endogenous hormones and breast cancer risk. Epidemiologic reviews. 1993;15(1):48-65.

626 52. Ho SM. Estrogen, progesterone and epithelial ovarian cancer. Reproductive biology and endocrinology : RB\&E. 2003;1:73.

628 53. Rizner TL. Estrogen biosynthesis, phase I and phase II metabolism, and action 629 in endometrial cancer. Molecular and cellular endocrinology. 2013;381(12):124-139.

631 54. Heldring N, Pike A, Andersson S, et al. Estrogen receptors: how do they signal and what are their targets. Physiological reviews. 2007;87(3):905-931.

633 55. Huang B, Warner M, Gustafsson JA. Estrogen receptors in breast 634 carcinogenesis and endocrine therapy. Molecular and cellular endocrinology. 2014.

636 56. Althuis MD, Fergenbaum JH, Garcia-Closas M, Brinton LA, Madigan MP, 637 Sherman ME. Etiology of hormone receptor-defined breast cancer: a 638 systematic review of the literature. Cancer Epidemiol Biomarkers Prev. 2004;13(10):1558-1568.

640 57. Goodwin PJ. Obesity and endocrine therapy: host factors and breast cancer outcome. Breast. 2013;22 Suppl 2:S44-47. 
642 58. Subbaramaiah K, Howe LR, Bhardwaj P, et al. Obesity is associated with 643 inflammation and elevated aromatase expression in the mouse mammary 644 gland. Cancer Prev Res (Phila). 2011;4(3):329-346.

645 59. Duncan RE, Ahmadian M, Jaworski K, Sarkadi-Nagy E, Sul HS. Regulation of 646 lipolysis in adipocytes. Annual review of nutrition. 2007;27:79-101.

647 60. Choe SS, Huh JY, Hwang IJ, Kim JI, Kim JB. Adipose Tissue Remodeling: Its Role 648 in Energy Metabolism and Metabolic Disorders. Front Endocrinol (Lausanne). $649 \quad 2016 ; 7: 30$.

650 61. Balaban S, Lee LS, Schreuder M, Hoy AJ. Obesity and Cancer Progression: Is 651 There a Role of Fatty Acid Metabolism? BioMed research international. 652 $2015 ; 2015: 274585$.

653 62. Henry SL, Bensley JG, Wood-Bradley RJ, Cullen-McEwen LA, Bertram JF, 654 Armitage JA. White adipocytes: more than just fat depots. The international 655 journal of biochemistry \& cell biology. 2012;44(3):435-440.

656 63. Suganami T, Tanaka M, Ogawa Y. Adipose tissue inflammation and ectopic lipid accumulation. Endocrine journal. 2012;59(10):849-857.

658 64. Geisler CE, Renquist BJ. Hepatic lipid accumulation: cause and consequence of 659 dysregulated glucoregulatory hormones. J Endocrinol. 2017.

660 65. Browning JD, Szczepaniak LS, Dobbins R, et al. Prevalence of hepatic steatosis 661 in an urban population in the United States: impact of ethnicity. Hepatology.

663 66. Bellentani S, Scaglioni F, Marino M, Bedogni G. Epidemiology of non-alcoholic 664 fatty liver disease. Dig Dis. 2010;28(1):155-161. 
665 67. White DL, Kanwal F, El-Serag HB. Association between nonalcoholic fatty liver disease and risk for hepatocellular cancer, based on systematic review. Clinical gastroenterology and hepatology : the official clinical practice journal of the American Gastroenterological Association. 2012;10(12):1342-1359 e1342.

669 68. Vanni E, Bugianesi E, Kotronen A, De Minicis S, Yki-Jarvinen H, Svegliati-Baroni G. From the metabolic syndrome to NAFLD or vice versa? Digestive and liver disease : official journal of the Italian Society of Gastroenterology and the Italian Association for the Study of the Liver. 2010;42(5):320-330.

673 69. Berardis S, Sokal E. Pediatric non-alcoholic fatty liver disease: an increasing 674 public health issue. European journal of pediatrics. 2014;173(2):131-139.

675 70. Tolman KG, Dalpiaz AS. Treatment of non-alcoholic fatty liver disease. Therapeutics and clinical risk management. 2007;3(6):1153-1163.

677 71. Farese RV, Jr., Zechner R, Newgard CB, Walther TC. The problem of establishing relationships between hepatic steatosis and hepatic insulin resistance. Cell metabolism. 2012;15(5):570-573.

680 72. Hui JM, Kench JG, Chitturi S, et al. Long-term outcomes of cirrhosis in nonalcoholic steatohepatitis compared with hepatitis C. Hepatology.

683 73. Smits MM, van Geenen EJ. The clinical significance of pancreatic steatosis. 684 Nature reviews Gastroenterology \& hepatology. 2011;8(3):169-177.

685 74. van Geenen EJ, Smits MM, Schreuder TC, van der Peet DL, Bloemena E, Mulder 686 CJ. Nonalcoholic fatty liver disease is related to nonalcoholic fatty pancreas 687 disease. Pancreas. 2010;39(8):1185-1190. 
688 75. Byrne AM, Bouchier-Hayes DJ, Harmey JH. Angiogenic and cell survival functions of vascular endothelial growth factor (VEGF). Journal of cellular and molecular medicine. 2005;9(4):777-794.

691 76. Cottam D, Fisher B, Ziemba A, et al. Tumor growth factor expression in obesity 692 and changes in expression with weight loss: another cause of increased 693 virulence and incidence of cancer in obesity. Surgery for obesity and related diseases : official journal of the American Society for Bariatric Surgery. 2010;6(5):538-541.

77. Iwaki T, Urano T, Umemura K. PAI-1, progress in understanding the clinical problem and its aetiology. British journal of haematology. 2012;157(3):291298.

699 78. Bauman KA, Wettlaufer SH, Okunishi K, et al. The antifibrotic effects of 700 plasminogen activation occur via prostaglandin E2 synthesis in humans and

79. Malik R, Lelkes PI, Cukierman E. Biomechanical and biochemical remodeling of stromal extracellular matrix in cancer. Trends in biotechnology. 2015;33(4):230-236.

705 80. Slaughter KN, Thai T, Penaroza S, et al. Measurements of adiposity as clinical 706 biomarkers for first-line bevacizumab-based chemotherapy in epithelial

708 81. Casagrande DS, Rosa DD, Umpierre D, Sarmento RA, Rodrigues CG, Schaan BD. 709 Incidence of cancer following bariatric surgery: systematic review and meta710 analysis. Obes Surg. 2014;24(9):1499-1509. 
711 82. Hursting SD, Dunlap SM, Ford NA, Hursting MJ, Lashinger LM. Calorie 712 restriction and cancer prevention: a mechanistic perspective. Cancer Metab. 2013;1(1):10.

714 83. Longo VD, Fontana L. Calorie restriction and cancer prevention: metabolic and 715 molecular mechanisms. Trends Pharmacol Sci. 2010;31(2):89-98.

716 84. Mattison JA, Roth GS, Beasley TM, et al. Impact of caloric restriction on health 717 and survival in rhesus monkeys from the NIA study. Nature. 2012;489(7415):318-321.

719 85. Colman RJ, Anderson RM, Johnson SC, et al. Caloric restriction delays disease 720 onset and mortality in rhesus monkeys. Science. 2009;325(5937):201-204.

721 86. Lv M, Zhu X, Wang H, Wang F, Guan W. Roles of caloric restriction, ketogenic 722 diet and intermittent fasting during initiation, progression and metastasis of cancer in animal models: a systematic review and meta-analysis. PLoS One. 2014;9(12):e115147.

725 87. Weiss EP, Racette SB, Villareal DT, et al. Improvements in glucose tolerance and insulin action induced by increasing energy expenditure or decreasing energy intake: a randomized controlled trial. Am J Clin Nutr. 2006;84(5):10331042.

729 88. Most J, Tosti V, Redman LM, Fontana L. Calorie restriction in humans: An $730 \quad$ update. Ageing Res Rev. 2016.

731 89. Fabian CJ, Kimler BF, Donnelly JE, et al. Favorable modulation of benign breast 732 tissue and serum risk biomarkers is associated with $>10 \%$ weight loss in 733 postmenopausal women. Breast Cancer Res Treat. 2013;142(1):119-132. 
734 90. Byers T, Sedjo RL. Does intentional weight loss reduce cancer risk? Diabetes $735 \quad$ Obes Metab. 2011;13(12):1063-1072.

736 91. Fontana L, Villareal DT, Das SK, et al. Effects of 2-year calorie restriction on 737 circulating levels of IGF-1, IGF-binding proteins and cortisol in nonobese men 738 and women: a randomized clinical trial. Aging Cell. 2016;15(1):22-27.

739 92. Saleh AD, Simone BA, Palazzo J, et al. Caloric restriction augments radiation $740 \quad$ efficacy in breast cancer. Cell Cycle. 2013;12(12):1955-1963.

741 93. Brandhorst S, Longo VD. Fasting and Caloric Restriction in Cancer Prevention $742 \quad$ and Treatment. Recent Results Cancer Res. 2016;207:241-266.

743 94. Mattson MP, Longo VD, Harvie M. Impact of intermittent fasting on health and $744 \quad$ disease processes. Ageing Res Rev. 2016.

745 95. Longo VD, Mattson MP. Fasting: molecular mechanisms and clinical 746 applications. Cell metabolism. 2014;19(2):181-192.

747 96. Harvie MN, Howell T. Could Intermittent Energy Restriction and Intermittent 748 Fasting Reduce Rates of Cancer in Obese, Overweight, and Normal-Weight 749 Subjects? A Summary of Evidence. Adv Nutr. 2016;7(4):690-705.

750 97. Harvie M, Howell A. Energy restriction and the prevention of breast cancer. $751 \quad$ Proc Nutr Soc. 2012;71(2):263-275.

752 98. Harvie MN, Pegington M, Mattson MP, et al. The effects of intermittent or 753 continuous energy restriction on weight loss and metabolic disease risk 754 markers: a randomized trial in young overweight women. Int J Obes (Lond). 755 2011;35(5):714-727. 
99. Sofi F, Cesari F, Abbate R, Gensini GF, Casini A. Adherence to Mediterranean diet and health status: meta-analysis. BMJ. 2008;337:a1344.

758 100. Brown T, Avenell A, Edmunds LD, et al. Systematic review of long-term lifestyle interventions to prevent weight gain and morbidity in adults. Obes Rev.

101. Romaguera D, Norat T, Mouw T, et al. Adherence to the Mediterranean diet is associated with lower abdominal adiposity in European men and women. $J$ Nutr. 2009;139(9):1728-1737.

764 102. Schwingshackl L, Hoffmann G. Adherence to Mediterranean diet and risk of cancer: an updated systematic review and meta-analysis of observational

767 103. de Lorgeril M, Salen P, Martin JL, Monjaud I, Boucher P, Mamelle N. 768 Mediterranean dietary pattern in a randomized trial: prolonged survival and possible reduced cancer rate. Arch Intern Med. 1998;158(11):1181-1187.

770 104. Harvie MN, Sims AH, Pegington $\mathrm{M}$, et al. Intermittent energy restriction 771 induces changes in breast gene expression and systemic metabolism. Breast $772 \quad$ Cancer Res. 2016;18(1):57.

773 105. Safdie FM, Dorff T, Quinn D, et al. Fasting and cancer treatment in humans: A $774 \quad$ case series report. Aging (Albany NY). 2009;1(12):988-1007.

775 106. de Groot S, Vreeswijk MP, Welters MJ, et al. The effects of short-term fasting 776 on tolerance to (neo) adjuvant chemotherapy in HER2-negative breast cancer patients: a randomized pilot study. BMC Cancer. 2015;15:652. 
107. Ballard-Barbash R, Friedenreich CM, Courneya KS, Siddiqi SM, McTiernan A, Alfano CM. Physical activity, biomarkers, and disease outcomes in cancer survivors: a systematic review. J Natl Cancer Inst. 2012;104(11):815-840.

108. Shalamzari SA, Agha-Alinejad H, Alizadeh S, et al. The effect of exercise training on the level of tissue IL-6 and vascular endothelial growth factor in breast cancer bearing mice. Iran J Basic Med Sci. 2014;17(4):231-258.

109. Jiang W, Zhu Z, Thompson HJ. Effects of physical activity and restricted energy intake on chemically induced mammary carcinogenesis. Cancer Prev Res (Phila). 2009;2(4):338-344.

110. Pischon T, Hankinson SE, Hotamisligil GS, Rifai N, Rimm EB. Leisure-time physical activity and reduced plasma levels of obesity-related inflammatory

111. Esposito K, Pontillo A, Di Palo C, et al. Effect of weight loss and lifestyle changes on vascular inflammatory markers in obese women: a randomized trial. JAMA. 2003;289(14):1799-1804.

112. Imayama I, Ulrich CM, Alfano CM, et al. Effects of a caloric restriction weight

797 113. Jones SB, Thomas GA, Hesselsweet SD, Alvarez-Reeves M, Yu H, Irwin ML. Effect of exercise on markers of inflammation in breast cancer survivors: the Yale exercise and survivorship study. Cancer Prev Res (Phila). 2013;6(2):109118. 
801 114. Kruk J, Czerniak U. Physical activity and its relation to cancer risk: updating 802 the evidence. Asian Pac J Cancer Prev. 2013;14(7):3993-4003.

803 115. Courneya KS, Sellar CM, Stevinson C, et al. Randomized controlled trial of the 804 effects of aerobic exercise on physical functioning and quality of life in 805 lymphoma patients. J Clin Oncol. 2009;27(27):4605-4612.

806 116. Nilsen TS, Raastad T, Skovlund E, et al. Effects of strength training on body 807 composition, physical functioning, and quality of life in prostate cancer 808 patients during androgen deprivation therapy. Acta Oncol. 2015;54(10):18058091813.

810 117. Schulz SV, Laszlo R, Otto S, et al. Feasibility and effects of a combined adjuvant 811 high-intensity interval/strength training in breast cancer patients: a single$812 \quad$ center pilot study. Disabil Rehabil. 2017:1-8.

813 118. Winningham ML, MacVicar MG. The effect of aerobic exercise on patient 814 reports of nausea. Oncol Nurs Forum. 1988;15(4):447-450.

815 119. MacVicar MG, Winningham ML, Nickel JL. Effects of aerobic interval training 816 on cancer patients' functional capacity. Nurs Res. 1989;38(6):348-351.

817 120. Courneya KS, Segal RJ, Mackey JR, et al. Effects of aerobic and resistance 818 exercise in breast cancer patients receiving adjuvant chemotherapy: a 819 multicenter randomized controlled trial. JClin Oncol. 2007;25(28):4396-4404. 820 121. Sprod LK, Mohile SG, Demark-Wahnefried W, et al. Exercise and Cancer 821 Treatment Symptoms in 408 Newly Diagnosed Older Cancer Patients. J Geriatr $822 \quad$ Oncol. 2012;3(2):90-97. 
823 122. Mishra SI, Scherer RW, Snyder C, Geigle PM, Berlanstein DR, Topaloglu O. 824 Exercise interventions on health-related quality of life for people with cancer 825 during active treatment. Clin Otolaryngol. 2012;37(5):390-392.

826 123. Kushi LH, Doyle C, McCullough M, et al. American Cancer Society Guidelines on 827 nutrition and physical activity for cancer prevention: reducing the risk of 828 cancer with healthy food choices and physical activity. CA Cancer J Clin. $829 \quad 2012 ; 62(1): 30-67$.

830 124. Paoli A, Rubini A, Volek JS, Grimaldi KA. Beyond weight loss: a review of the 831 therapeutic uses of very-low-carbohydrate (ketogenic) diets. Eur J Clin Nutr. $832 \quad$ 2013;67(8):789-796.

833 125. Fu SP, Li SN, Wang JF, et al. BHBA suppresses LPS-induced inflammation in BV8342 cells by inhibiting NF-kappaB activation. Mediators Inflamm. $835 \quad 2014 ; 2014: 983401$.

836 126. Goldberg EL, Asher JL, Molony RD, et al. beta-Hydroxybutyrate Deactivates 837 Neutrophil NLRP3 Inflammasome to Relieve Gout Flares. Cell Rep. $838 \quad$ 2017;18(9):2077-2087.

839 127. Merra G, Gratteri S, De Lorenzo A, et al. Effects of very-low-calorie diet on body 840 composition, metabolic state, and genes expression: a randomized double841 blind placebo-controlled trial. Eur Rev Med Pharmacol Sci. 2017;21(2):329$842 \quad 345$.

843 128. Woolf EC, Curley KL, Liu Q, et al. The Ketogenic Diet Alters the Hypoxic 844 Response and Affects Expression of Proteins Associated with Angiogenesis, 
Invasive Potential and Vascular Permeability in a Mouse Glioma Model. PLoS One. 2015;10(6):e0130357.

847 129. Nandivada P, Fell GL, Pan AH, et al. Eucaloric Ketogenic Diet Reduces Hypoglycemia and Inflammation in Mice with Endotoxemia. Lipids.

850 130. Badman MK, Kennedy AR, Adams AC, Pissios P, Maratos-Flier E. A very low 851 carbohydrate ketogenic diet improves glucose tolerance in ob/ob mice

854 131. Tisdale MJ, Brennan RA, Fearon KC. Reduction of weight loss and tumour size 855 in a cachexia model by a high fat diet. Br J Cancer. 1987;56(1):39-43.

856 132. Allen BG, Bhatia SK, Anderson CM, et al. Ketogenic diets as an adjuvant cancer 857 therapy: History and potential mechanism. Redox Biol. 2014;2:963-970.

858 133. Branco AF, Ferreira A, Simoes RF, et al. Ketogenic diets: from cancer to mitochondrial diseases and beyond. Eur J Clin Invest. 2016;46(3):285-298.

860 134. Wakefield AP, House JD, Ogborn MR, Weiler HA, Aukema HM. A diet with 35\% 861 of energy from protein leads to kidney damage in female Sprague-Dawley rats. 862 BrJ Nutr. 2011;106(5):656-663.

863 135. Ellenbroek JH, van Dijck L, Tons HA, et al. Long-term ketogenic diet causes 864 glucose intolerance and reduced beta- and alpha-cell mass but no weight loss 865 in mice. Am J Physiol Endocrinol Metab. 2014;306(5):E552-558. 


\section{$867 \quad$ Figure Legends}

868 Figure 1: Obesity is associated with increased risk of developing and dying from the

869 following cancers: breast (in postmenopausal women), ovarian, liver, gallbladder, kidney

870 (renal cell), colon, pancreatic, gastric, esophageal (adenocarcinoma), endometrial,

871 thyroid, multiple myeloma, and meningioma ${ }^{4}$. In addition, obesity is associated with

872 progression (but not incidence) of prostate cancer $^{8}$.

873

874 Figure 2: Obesity and metabolic syndrome result in many metabolic disturbances

875 including elevations in circulating insulin, adipokines (e.g. leptin-to-adiponectin ratio),

876 cytokines, angiogenic factors (PAI-1 and VEGF), as well as increased prevalence of

877 adipose tissue crown-like structures, a marker of adipose inflammation. These factors can

878 activate receptor tyrosine kinase signaling through the PI3 kinase (PI3K)/Akt/mammalian

879 target of rapamycin (mTOR) pathway. An increase in steady state signaling through this

880 pathway can drive increases in cellular proliferation and protein translation, and reinforce

881 cancer-associated metabolic reprogramming. Activation of NF- $\kappa B$ by proinflammatory

882 cytokines, induces translocation to the nucleus and upregulates expression of genes

883 involved in survival proliferation, inflammation and immune regulation. Together,

884 obesity-associated elevation of growth factor signaling and inflammation and reduction

885 of vascular integrity fosters a microenvironment favorable for tumorigenesis, increasing

886 cancer risk and progression.

887

888 Figure 3: The human body contains two types of adipocytes: white adipocytes (which

889 have a unilocular lipid droplet) and brown adipocytes (which have many small lipid 
890 droplets). When engorged with triglyceride, white adipocytes secrete a number of factors

891 that promote growth factor signaling and inflammation including leptin, resistin, insulin-

892 like growth factor (IGF)-1, free fatty acids, tumor necrosis factor (TNF)- $\alpha$ and

893 interleukin (IL)-6. Additionally, they reduce production of anti-inflammatory

894 adiponectin. Brown adipocytes secrete several factors involved in thermogenesis,

895 decreased inflammation, normalized insulin sensitivity and/or increased energy

896 expenditure such as adiponectin, bone morphogenetic proteins, neuregulin-4, lactate,

897 triiodothyronine (T3), retinaldehyde, and fibroblast growth factor (FGF)-21.

898

899 Figure 4: In obesity, as adipocytes accumulate triglycerides, their secretomes shift

900 towards the production of pro-inflammatory and/or insulin resistant molecules, including

901 numerous cytokines, adipokines (e.g. leptin and resistin), and free fatty acids.

902 Engorged/necrotic adipocytes attract macrophages and other immune cells that further

903 contribute to the pro-inflammatory environment. The altered secretome resulting from

904 macrophage-adipocyte interactions modulates several enzymes associated with increased

905 inflammation-related lipid mediators (such as prostaglandins and leukotrienes) and

906 hormones (such as aromatase, which converts androgens to estrogens).

907

908 Figure 5: Dietary and lifestyle interventions of caloric restriction (CR), intermittent

909 fasting (IF), physical activity (PA) and adherence to a ketogenic diet (KD), have been

910 shown to reduce adiposity and favorably modulate many of the same cancer risk

911 biomarkers that are impacted by obesity including: insulin, IGF-1, leptin, adiponectin,

912 cytokines, angiogenic factors, and crown-like structures. These metabolic alterations 
913 could result in downstream reductions in growth factor signaling, inflammation, and

914 angiogenesis and attenuate cancer risk and progression. Metabolic alterations with CR, IF

915 and PA interventions have been associated with reduced cancer risk and progression.

916 While KD has not been linked to cancer risk, it has been demonstrated that adherence to

917 KD reduces cancer risk and progression in preclinical studies. a) Insufficient evidence

918 exist to conclude the impact of PA and IF on PAI-1 and VEGF expression. b) Current

919 literature does not exist examining the impact of KD on crown-like structures. 